\title{
La Littérature vietnamienne entre modernité et traditions: étude des romans Celui qui règnera et Frères de sang de Pham Van Ky.
}

Souleymane FOFANA

\section{Introduction}

\section{$\mathrm{A}$}

l'instar de beaucoup de pays d'Afrique, le Vietnam a subi pendant de très longues années la colonisation française. Celle-ci a non seulement bouleversé les structures sociales et politiques de ce petit pays de l'Asie mais aussi et surtout a influencé le mode de pensée notamment en ce qui concerne la littérature. Pham Van Ky, écrivain vietnamien qui a séjourné en France depuis 1938 jusqu'à sa mort, à travers deux récits fictionnels, de belle facture a abordé, dans les romans: Frères de sang et Celui qui règnera quelques problèmes auxquels la société vietnamienne est confrontée. On peut citer, entre autres, l'éternelle opposition entre modernité et traditions.

Dans le cadre de notre analyse, nous avons décidé de plancher sur ce sujet. Et ce, pour une raison principale. En effet, réfléchir sur le thème : "Modernité et traditions" revient également à examiner les rapports entre colonisateur et colonisé.

L'Occident, au nom de sa "prétendue" mission civilisatrice, au nom également de la modernité n'a-t-il pas fait table rase des traditions millénaires des pays colonisés ? C'est pourquoi, l'intérêt majeur de ces deux romans est qu'ils montrent avec précision "la physionomie du colonisé" et donne de précieuses informations sur le drame de sa situation. Il met également en exergue la conduite du colonisateur et du colonisé et les rapports qui les liaient l'un à l'autre. En effet, le couple "colonisé- 
colonisateur," bien qu'étant antinomique reste cependant lié par une communauté de destin car "sans colonisé, il n'y a pas de colonisateur et sans colonisateur, il n'y a pas de colonisé."1 Cela peut s'appliquer également au couple "traditions-modernité," objet de notre réflexion. Comment peut-on croire au confucianisme, au culte des ancêtres, au taoisme, à l'animisme et intégrer les valeurs occidentales qui n'accordent aucune importance à ces croyances citées ci-dessus ? En d'autres termes, comment modernisme et traditions peuvent-ils cohabiter ? Ou encore estil possible d'appartenir, à la fois, sans perdre son identité, à la culture du colonisé et à celle du colonisateur? Telles sont quelques unes des questions auxquelles nous aurons à répondre dans notre analyse.

Notre présente étude s'articulera autour de quatre points. De prime abord, nous allons définir les thèmes de colonisation et d'identité qui reviennent, de façon récurrente, dans la littérature vietnamienne. Ensuite, nous allons essayer d'établir un rapport entre ces deux concepts. Dans la deuxième partie de notre travail, nous allons suivre le même schéma en ce qui concerne le couple "Modernité et traditions." Pour clore, nous analyserons en quoi les romans Frères de sang et Celui qui règnera peuvent être considérés comme l'expression de l'identité culturelle du peuple vietnamien. Cela revient à dégager la signification politique, sociale, religieuse, économique et la portée sémantique de ces deux œuvres.

Notre démarche pour étudier les romans soumis à notre investigation va s'appuyer sur la socio-critique, méthode qui a l'avantage de tenir compte de l'environnement socio-culturel dans la compréhension des textes littéraires. Ensuite, nous utiliserons la méthode de commentaire et d'explication de textes pour mieux saisir le sens du discours de l'auteur.

\section{Colonisation et identité}

\section{Définition de la colonisation}

Que signifie colonisation? Il est difficile de donner une définition de ce concept qui satisfasse tout le monde car le mot "colonisation" est connoté idéologiquement. Un Belge ou un Français de l'administration dont les pays ont eu des colonies ne définirait pas le mot colonisation de la même manière qu'un Algérien, un Vietnamien ou un Sénégalais qui eux, ont subi l'action de la colonisation. Pour les premiers, la colonisation pourrait être perçue comme l'action de civiliser des peuples dits " non évolués." Pour les seconds, c'est le fait de s'accaparer par la force des richesses d'un pays, de pervertir la culture de ce peuple. Mais aujourd'hui, beaucoup d'intellectuels s'accordent à dire que l'entreprise coloniale a contribué à la dépersonnalisation du colonisé et à son acculturation. 
C'est cet aspect de la colonisation qui nous intéresse et que nous allons définir en nous appuyant sur les idées d'écrivains comme Jean-Paul Sartre, Frantz Fanon, Albert Memmi, Aimé Césaire...

Pour Frantz Fanon, le monde colonisé, à l'image de l'ex-mur qui divisait l'Allemagne en deux (l'Allemagne de l'ouest et l'Allemagne de l'est ) est "compartimenté." En témoigne cet extrait d'un passage tiré dans le livre Les damnés de la terre:

Le monde colonial est un monde compartimenté. Sans doute, est-il superflu, sur le plan de la description, de rappeler l'existence de villes indigènes et de villes européennes, d'écoles pour indigènes et d'écoles pour Européens, comme il est superflu de rappeler l'apartheid en Afrique du Sud... Le monde colonisé est un monde coupé en deux. La ligne de partage, la frontière en est indiquée par les casernes et les postes de police. Aux colonies, l'interlocuteur valable et institutionnel du colonisé, le porteparole du colon et du régime d'oppression est le gendarme ou le soldat. $^{2}$

De ce point de vue, on peut dire avec Frantz Fanon que la colonisation s'appuie sur des thèses "d'exclusion" pour asseoir des mécanismes d'oppression qui visent à dépouiller le colonisé de tous ses droits. C'est la raison pour laquelle Jean-Paul Sartre dans la préface du livre Portrait du colonisé d'Albert Memmi écrit:

Le colonialisme refuse les droits de l'homme à des hommes qu'il a soumis par la violence, qu'il maintient de force dans la misère et l'ignorance, donc, comme dirait Marx, en état de "soushumanité”. Dans les faits eux-mêmes, dans les institutions, dans la nature des échanges et de la production, le racisme est inscrit [...] l'indigène est un sous-homme, la Déclaration des Droits de l'Homme ne le concerne pas; inversement, puisqu'il n'a pas de droits, il est abandonné sans protection aux forces inhumaines de la nature, aux "lois d'airain" de l'économie. ${ }^{3}$

En d'autres termes, la colonisation aliène la personnalité du colonisé et le pose comme "objet." Il ne sera jamais acteur de l'histoire puisqu' on ne lui reconnaît pas d'identité. Il est né pour subir et non pas agir.

Dans l'Amant et Un barrage contre le Pacifique de Marguerite Duras, les personnages vietnamiens apparaissent comme des "ombres." On ne 
connaît ni leur origine, ni leur vie passée, encore moins le but de leurs actions dans les oeuvres citées ci-dessus. D'ailleurs, Marguerite Duras, n'a même pas jugé utile d'attribuer des noms à beaucoup d'entre eux.

Le lecteur d'Un barrage contre le Pacifique se rend compte que les enfants victnamiens meurent comme des mouches et les femmes sont des " usines" à fabriquer des enfants. Il y a une tendance à l'abstraction et à l'anonymat qui dénote du mépris du narrateur pour ces "personnages" qui apparaissent comme de "trop" dans la narration. Le passage suivant tiré de l'Amant de la Chine du Nord est symptomatique de ce état de fait: Le chinois demande d'où vient Thanh. Elle dit que la mère l'a trouvé en haut de la montagne à la frontière entre le Siam et le Cambodge un soir en revenant des poivrières avec des enfants. (106)

Ce qui nous amène à nous interroger sur l'identité des colonisés. De prime abord, que recouvre ce concept?

\section{Définition de l'identité}

Selon le dictionnaire Larousse, l'identité est le fait qu'une chose est de même nature qu'une autre. Autrement dit, c'est l'ensemble des circonstances qui font qu'une personne est bien telle personne déterminée. En effet, refléchir sur le problème de l'identité, c'est poser la question qui suis-je? Cela revient également à plancher sur la question de l'existence humaine: un sujet qui à première vue paraît simple, mais qui en réalité ne l'est pas. Le débat sur l'existence, depuis la période des Socrate, Platon, Aristote (phiolosophes grecs) jusqu'au vingt-et-unième siècle avec des écrivains comme Jean-Paul Sartre, Frantz Fanon, Aimé Césaire... continue et est très loin d'être épuisé. Cela pour dire que la difficulté de l'étude de ce concept s'explique par le fait qu'il touche l'homme dans ce qu'il a de plus profond à savoir son essence, son moi... en un mot, c'est ce qui fait que Paul est différent de Pierre, le Vietnamien du Français, l'Américain du Bosniaque, l'Africain du Tchétchène... Cette identité n'est pas figée. Elle peut s'enrichir ou se pervertir au contact d'une autre culture. Dans le roman Frères de sang de Pham Van Ky, on voit bien que le narrateur et personnage principal, après un séjour de dix ans en France, se sent totalement étranger. Il ne se reconnaît plus aux siens et ceux-ci également ne se reconnaissent plus en lui. À tel point qu'il se pose la question suivante: "Aurais-je changé à ce point ou étais-je seul à susciter, à préparer mon dépaysement" (14). Il en vient à la conclusion suivante: "Je ne suis qu'un étranger" (15).

En effet, déchiré entre la culture Occidentale et la Vietnamienne, le héros-narrateur vit une véritable angoisse existentielle. Il se sent, après 
plusieurs années passées en Europe, plus "Français" que Vietnamien. Cependant, il ne renie pas ses valeurs culturelles vietnamiennes. Et il justifie son retour actuel par un serment ancien qui le lie à Lê Tâm :

Pourtant, je ne serais pas venu au chevet du lit d'agonie de Mère. En déhors de ce serment ancien qui me liait à Lê Tâm, rien ne m'aurait déterminé à quitter la France; pas même une femme qui comblerait ma vie. (19)

Le roman Frères de sang met en exergue le choc des cultures et la difficulté à transposer ou à transférer les valeurs culturelles de civilisations d'un continent à un autre sans chambourler l'ordre établi. Or la colonisation avait pour but de donner une nouvelle identité aux colonisés. En effet, l'un des objectifs de la colonisation française visait l'assimilation des colonisés. C'est en ce sens qu'on enseignait dans les écoles des colonies l'histoire de la France et on faisait même croire que "les ancêtres des colonisés étaient des Gaulois." Les Vietnamiens, les Africains, les Algériens, les Français... avaient le même ancêtre: les Gaulois. Le roman Celui qui règnera atteste de cette situation:

À Hué, le traité de 1884 était signé depuis quatre ans. Dans les nouvelles écoles, des bambins à la tête rasée, détournés de leurs traditionnels Maîtres de caractères, ânonnaient avec conviction: Nos ancêtres, les Gaulois... On trichait avec son sang. On trichait partout, avec tout. On trichait. (28-9)

À ce propos, Frantz Fanon dans son livre Les damnés de la terre écrit fort justement:

Le colon fait l'histoire et sait qu'il la fait. Et parce qu'il se refère constamment à l'histoire de sa métropole, il indique en clair qu'il est ici le prolongement de cette métropole. L'histoire qu'il écrit n'est pas l'histoire du pays qu'il dépouille mais l'histoire de sa nation en ce qu'elle écume, viole et affame. L'immobilité à laquelle est condamnée le colonisé ne peut être remise en question que si le colonisé décide de mettre un terme à l'histoire de la colonisation, à l'histoire du pillage, pour faire exister l'histoire de la nation, l'histoire de la décolonisation. ${ }^{4}$ 
Après la lecture de cette réflexion de Frantz Fanon, la question que l'on peut se poser est la suivante: quel est le rapport entre colonisation et identité?

\section{Rapport identité et colonisation}

Frantz Fanon, dans son livre Les damnés de la terre définit les rapports entre colonisation et identité en ces termes:

Sur le plan de l'inconscient, le colonialisme ne cherchait donc pas à être perçu par l'indigène comme comme une mère douce et bienvaillante qui protège l'enfant d'un environnement hostile, mais bien sous la forme d'une mère qui sans cesse, empêche un enfant fondamentalement pervers de réussir son identité, de donner libre cours à des instincts maléfiques. La mère coloniale défend l'enfant contre lui-même, contre son moi, contre sa physiologie, sa biologie, son malheur ontologique. (154)

Ici, on voit bien que le rapport entre colonisation et identité est perverti puisque le colonisateur ne cherche pas à établir des rapports d'égalité avec le colonisé, mais plutôt à le prendre en charge comme un enfant. Il nie le "moi" du colonisé et cherche à lui substituer un autre "moi" qui est plus proche de ses fantasmes et de sa vision du monde. C'est ce que Jean-Paul Sartre souligne dans la préface du livre d'Albert Memmi Portrait $d u$ colonisé $e^{5}$ en ces termes:

Memmi a remarquablement décrit la suite de démarches qui les conduit à "l'auto-absolution." Le conservatisme entraîne la sélection des médiocres. Comment peut-elle fonder ses privilèges, cette élite d'usurpateurs conscients de leur médiocrité ? Un seul moyen: abaisser le colonisé pour se grandir, refuser la qualité d'homme aux indigènes, les définir comme de simples privations [...]. Le colon ne peut s'absoudre qu'en poursuivant systématiquement la "déshumanisation" du colonisé. (27)

Mais au-delà des rapports de force qui évidemment sont à l'avantage du colonisateur, il faut également souligner que ce dernier subit des influences du sujet colonial qui dénaturent sa personnalité et font de lui un éternel assisté. Autrement dit, colonisateur et colonisé entretiennent sans le vouloir un rapport dialectique qui fait que l'identité de l'un est influencée par l'autre et vice versa. C'est ce qu'Albert Memmi a su bien traduire 
dans son livre Portrait du colonisé en ces termes: "La colonisation fabrique des colonisés comme elle fabrique des colonisateurs."

Qu'en est-il maintenant de la situation du colonisé dans Frères de sang et Celui qui règnera de Pham Van Ky? D'entrée de jeu, il faut souligner que le conflit entre modernité et traditions dans les romans Frères de sang et Celui qui règnera n'est pas décrit en termes de violence entre colonisé et colonisateur mais il se traduit par l'opposition entre des valeurs de civilisations: celles du confucianisme (culte des ancêtres et de l'empereur) le bouddhisme (religion de la majorité des Vietnamiens), la piété filiale, un principe fondamental du confucianisme, et toutes ces croyances qui tirent leur source dans les coutumes millénaires vietnamiennes transmises de générations en générations et le modernisme prôné par le narrateur et personnage principal qui a vécu dix ans à l'extérieur et qui ne voit plus le monde de la même manière que ses compatriotes restés au pays. Le narrateur vit une véritable angoisse existentielle qui se traduit dans tous les actes de la vie par l'opposition entre modernité et traditions.

\section{Rapport modernité et traditions dans les romans Frères de sang et Celui qui règnera}

\section{Définition de la modernité}

Dans les romans Frères de sang et Celui qui règnera, la modernité est souvent opposée à la tradition. La modernité prônée par l'étudiant vietnamien qui a séjourné dix ans en France peut se définir comme tout ce qui convient au temps présent ou à une époque relativement récente. D'abord, l'étudiant vietnamien, une fois rentré dans son pays veut briser les barrières de classes qui régissent la société vietnamienne. Il élargit le droit des domestiques, accepte en tant que fils de mandarin de travailler pour mériter son salaire et révolutionne le système d'irrigation. En témoigne ce passage extrait dans le roman Celui qui règnera: "Et pour lui montrer qu'avant tout, j'ambitionnais de soulager le sort des déshérités, j'appelai Tu, Sau, et Bay : 3/4 Vous serez inscrits, leur dis-je, sur les rôles d'impôts, exonérés des corvées et prestations de toutes sortes. Et vous aurez voix consultative dans les affaires de la commune" (74). Et plus loin, il fait remarquer: "Nous diviserons la récolte en huit parts, au lieu d'en prélever la moitié pour payer les redevances. Et je travaillerai au même titre que vous autres pour mériter mon salaire" (75-6). Il poursuit: "Notre système d'irrigation, poursuivis-je ne me semble pas des plus perfectionnés. Nous construisons une noria actionnée par un courant... de la rivière" (76). 
La modernité dans le roman Celui qui règnera est l'introduction de nouvelles valeurs susceptibles d'améliorer les conditions de vie des populations vietnamiennes. Elle intègre l'idée de progrès puisque la construction d'une noria actionnée par un courant de la rivière vise à augmenter la production. La modernité dans le roman Celui qui règnera, c'est également l'intauration de plus de justice sociale. En décidant d'accorder les mêmes droits aux domestiques qu'aux mandarins, le narrateur et personnage principal s'attaque ici au fondement social et moral de la société vietnamienne. Celle-ci est basée sur les différences de classes et une organisation sociale fondée sur le confucianisme, la piété filiale,... en un mot, sur les coutumes transmises de générations à générations. Que recouvre maintenant le concept de traditions ?

\section{Définition de la tradition}

La tradition peut être comprise comme la transmission de doctrines, de légendes, de coutumes... pendant un long espace de temps, spécialement par la parole et par l'exemple. Dans les romans Frères de sang et Celui qui règnera, la tradition du confucianisme, de la piété filiale survit grâce à la transmission des coutumes de génération en génération.

Dans le roman Frères de sang, l'étudiant vietnamien, après un séjour de dix ans à l'étranger se rend compte dès son retour dans son pays de l'immuabilité de la tradition. Juste après son retour, il fait le constat suivant

Aucun changement de dynastie, aucun bouleversement politique, n'auraient pu modifier la structure de ma famille. Son éthique ramenait tout à un sentiment unique: la piété filiale. L'enfant le doit à ses descendants car c'est d'eux qu'il reçoit la vie. Le peuple le doit au mandarin "Père et Mère" car de lui, il reçoit l' apanage du roi. Le mandarin le doit au roi, car de lui, il reçoit la faveur du ciel... Le village lui-même, personne morale ayant son libre arbitre, la responsabilité entière de ses actes, petits États dans l'État, fonctionnant sous un gouvernement oligarchique, constituait une redoutable inexpugnable, une cellule-témoin, inaccessible à toute réforme, un modèle de la pérennité. (25-26)

Alors que la tradition se caractérise par son immuabilité, la modernité, quant à elle, se définit comme une perpétuelle remise en cause. Le premier est tourné vers le passé, le second vers le futur. Comment ce couple 
antinomique par nature fonctionne dans Frères de sang et Celui qui règnera?

\section{Conflit modernité et traditions}

Dans les romans Frères de sang et Celui qui règnera, les concepts de modernité et traditions fonctionnent par opposition. Ils s'excluent mutuellement. L'un ne peut s'accomoder de la présence de l'autre et vice versa. Un dialogue dans le roman Celui qui règnera atteste de cette situation:

- Pour quand l'érection du pagodon? demanda Ong Hai, impatient.

-Demain? suggéra Tu. Ne serait-ce que pour le choix du terrain.

- Pas de géomancien! Criai-je à la surprise de tous...

L'oncle-maire sursauta à ce mot, mais il se contenta de ricaner...

L'assemblée éclata de rire. (79)

Ici, on voit bien que l'étudiant vietnamien est le symbole de la modernité et son oncle-maire, celui des valeurs traditionnelles. Dans Frères de sang et Celui qui règnera, l'affrontement entre ces deux personnages est permanent.

Les deux romans de Pham Van Ky que nous étudions présentement rappellent étrangement un autre roman: L'Aventure ambigü̈ $\ddot{C}^{7} \mathrm{de} C$ Cheikh Hamidou Kane. Dans ce roman, le conflit entre la modernité et la tradition avait pris des proportions inquiétantes. Concernant la question de la scolarisation des enfants diallobé, un dilemne s'était posé: certaines personnages du roman estimaient que le fait d'envoyer les "Diallobé" à l'école Occidentale allait leur faire perdre leur identité africaine. La Grande Royale, tout comme l'étudiant vietnamien dans le roman Celui qui règnera avait opté pour le modernisme. Elle avait justifié sa position en ces termes:

Notre grand-père, en même temps que tous les habitants de ce pays a été réveillé un matin par une clameur qui montait du fleuve. [...] Son coeur était intrépide et il attachait plus de prix à la liberté qu'à la vie... Notre grand-père ainsi que son élite ont été défaits. Pourquoi ? Comment ? Les nouveaux venus, seuls le savent [...]. (47)

C'est le même exemple que veut suivre l'étudiant vietnamien dans le roman Celui qui règnera. Il fait remarquer que la civilisation occidentale 
n'est pas meilleure, mais elle a su s'imposer au monde entier: "Ces imbéciles ignorent également qu'en 1894, dans la guerre de Corée, les Nippons triomphèrent de la Chine avec les armes de l'ouest" (85).

L'objectif du narrateur et personnage principal n'est pas de bouleverser l'ordre établi de sa société mais d'introduire de judicieuses reformes qui vont améliorer les conditions de vie de ses concitoyens. C'est en ce sens que Frères de sang et Celui qui règnera peuvent être perçus comme l'expression d'une identité culturelle.

\section{Frères de sang et Celui qui règnera : expression d'une identité culturelle?}

1. Le concept d'engagement dans la littérature vietnamienne.

De prime abord, que signifie le thème de l'engagement ?

L'engagement, selon le dictionnaire Larousse, peut être défini comme "le fait de prendre position dans les luttes sociales et politiques de son temps." Elle s'oppose au non-engagement qui consiste pour l'écrivain à s'enfermer dans sa tour d'Ivoire et à se désintéresser du sort de ses contemporains. Frantz Fanon, dans son livre Les damnés de la terre ${ }^{8}$ définit la mission de l'écrivain en ces termes:

L'homme colonisé qui écrit pour son peuple quand il utilise le passé doit le faire dans l'intention d'ouvrir l'avenir, d'inviter à l'action, de fonder l'espoir. Mais pour assurer l'espoir, pour lui donner densité, il faut participer à l'action, s'engager corps et âme dans le combat national. On peut parler de tout mais quand on décide de parler de cette chose unique dans la vie d'un homme que représente le fait d'ouvrir l'horizon, de porter la lumière chez soi-même et son peuple, alors il faut musculairement collaborer. (170)

Dans les romans Frères de sang et Celui qui règnera, l'engagement de l'écrivain est plus culturel qu'idéologique. Celui-ci se contente uniquement d'opposer les valeurs de la civilisation vietnamienne basee sur le confucianisme (culte des ancêtres et de l'empereur), la piété filiale, le boudhisme, religion pratiquée au Vietnam et les Occidentales basées sur le rationalisme et la chrétienneté. Frères de sang et Celui qui règnera sont deux récits fictionnels racontés par un jeune Vietnamien, personnage principal du récit. C'est un narrateur intra- et homo-diégétique puisqu'il est narrateur et personnage principal de son propre discours. Il est 
omniscient puisqu'il lit dans la pensée de ses personnages et c'est à travers son regard que le lecteur peut suivre le déroulement du récit. En effet, après avoir séjourné plus de dix ans en France, le héros-narrateur, de retour dans son pays, nous fait découvrir, à travers son regard, la société vietnamienne aussi bien de l'intérieur que de l'extérieur. Tout le récit se déroule sous forme d'oppositions dynamiques: Occident / Orient; individu /communauté; obéissance/ révolte; pureté / corruption; rêve / réalité; tradition / modernité; paroles / silence; extérieur / intérieur; mariage / obsèques; puissance / impuissance; action / inaction; jour / nuit ... Ces oppositions, contrairement à ce qu'on pourrait penser, sont plus complexes que binaires. Le héros-narrateur est au centre de toutes ces oppositions qui sont en réalité l'expression de l'identité culturelle vietnamienne. Celleci se caractérise par sa complexité et son dynamisme.

\section{Le refus des valeurs occidentales}

Les romans Frères de sang et Celui qui règnera mettent en exergue le choc des cultures et la difficulté à transposer ou à transférer les valeurs de civilisations d'un continent à un autre sans chambourler l'ordre établi. En effet, l'étudiant vietnamien, dans le roman Frères de sang quand il montre son diplôme ès lettres de l'université de Paris à son père, celui-ci s'empresse de demander: “À quel grade mandarinal es-tu assimilé?" Et le fils de répondre: “À aucun Père." Le Père de renchérir: “On ne t'a pourtant pas délivré cette distinction pour rien" (31).

Dialogue de sourds, choc de cultures, rejet de la civilisation occidcntale, tout dans le roman indique que la guerre culturelle entre l'Occident et l'Orient est plus "meurtrière," plus intense que celle qui a réellement opposé l'armée française et les troupes vietnamiennes.

Le roman Celui qui règnera peut être considéré comme la suite logique de Frères de sang. On retrouve les mêmes thèmes dans les deux romans, le même narrateur, le même récit, le même espace, et les mêmes personnages. Dans le roman Celui qui règnera on voit que la quête du modernisme de l'étudiant vietnamien qui se manifeste par l'introduction de nouvelles valeurs dans le microcosme social et culturel du Vietnam va se heurter à maintes difficultés. L'oncle-maire considère son neveu comme un anarchiste. Il s'en prend au cours d'une réunion publique à ce dernier en ces termes :

Nous, les fils du dragon et neveux des fées, nous vivons dans le respect des traditions, dans la crainte des Esprits... Mais, voici un an, surgit ce révolutionnaire (il me désigna du doigt, 
formé à l'école de l'Occident. Il pénétra dans le village, il disloqua le cortège des funérailles de sa mère, il ne se plia au Rite qu'à mes objurgations réitérées, il dispersa peu après les reliques de son père entre les mains de ses laquais, il projeta de redistribuer les terres, il déchira les reconnaissances de dettes, il entreprit de transformer en palais les masures de ses débiteurs, il insulta ses beaux-parents, les préfets de notre circonscription, il renia avec fracas, la femme que sa pauvre mère lui avait destinée, la violenta, la frappa..."(203)

Ici, l'étudiant est le symbole de l'Occident. L'oncle-maire reproche à son neveu de s'être occidentalisé et de renier ses propres valeurs culturelles.

\section{L'écriture comme moyen de libération}

Pham Van Ky raconte sa propre histoire à travers un retour imaginé. Dès lors, on peut se demander le but de cette oeuvre si l'histoire racontée n'est que pure invention. D'abord, il faut souligner que l'acte d'écrire se justifie par lui-même puisque la littérature est d'abord et avant tout un acte de liberté. C'est également une manière de créer une instabilité en posant des problèmes susceptibles de changer l'ordre normal des choses. Pham Van Ky, qui a vécu pendant plusieurs années en Occident utilise l'écriture comme une manière de se souvenir et de se guérir puisqu'il a souffert, dans sa chair, d'être exilé, pendant de si longues années.

L'écriture, de ce point de vue, a une vertu thérapeutique dans la mesure où elle permet d'acquérir la force pour affronter la dureté de l'existence. En écrivant, Pham Van Ky refoule toutes les frustrations enfouies dans son inconscient. Il nous fait également revivre son propre dépaysement. C'est pourquoi Linda Lê, ' écrivaine Vietnamienne, au cours d'un entretien accordé en avril 1999 au magazine littéraire "Lire," soutient "qu'écrire, c'est s'exiler." À la question du journaliste de Lire: "Auriezvous écrit si vous n'aviez pas quitté votre pays natal?" elle répond:

Oui, mais d'autres livres. Ecrire, c'est s'exiler. En écrivant, vous n'avez plus de toit, juste le ciel comme abri et c'est cette nudité devant les choses que vous aimez. Un écrivain ne peut écrire qu'en se sentant un enfant, un bâtard. Etre le fils de personne, d'aucune patrie, c'est pour moi la seule attitude possible. Je crois que l'on ne reste en vie que si l'on manifeste un désir de résistance à tout très ancré en soi. Une résistance à tout ce qui 
ne vous paraît pas relever de la beauté, de la vérité. Je refuse de faire cause commune. (32)

Au total, l'acte d'écrire se justifie par le fait qu'il permet de créer une instabilité et comme le dit si bien Charles Baudelaire, cité par Evelyne Cosset dans le livre intitule Les Fleurs du Mal"10: "La poésie permet d'atteindre un désir insatiable d'illimité" (35). Le langage utilisé dans les deux romans est très poétique, ce qui dénote l'importance de la poésie dans la culture vietnamienne. Ensuite, Frères de sang et Celui qui règnera sont des romans adressés à un public occidental. De ce point de vue, l'écriture devient facteur de libération puisqu'elle permet de réhabiliter les valeurs culturelles des peuples colonisés. À ce propos, Aimé Césaire ${ }^{11}$ affirmait dans le Cahier d'un retour au pays natal: "Et si je ne sais que parler, c'est pour vous que je parlerai. Ma bouche sera la bouche des malheurs qui n'ont point de bouche, ma voix, la liberté de ceux qui s'affaisent au cachot du désepoir" (9).

L'une des missions de la colonisation n'était-elle pas d'apporter la civilisation aux peuples dits "non évolués" ? En présentant les valeurs culturelles, politiques, et sociales de son pays, Pham Van Ky veut indirectement montrer que la colonisation a utilisé de fallacieux arguments pour agresser les peuples colonisés. En d'autres termes, avant l'arrivée de l'Occident, le Vietnam n'était pas désert de culture. Loin s'en faut! Les romans Frères de sang et Celui qui règnera nous montrent le dynamisme de la culture de cette partie de l'Asie. En ce sens, on peut dire que ce sont des romans engagés culturellement même si l'écrivain ne prône pas l'usage de la violence contre le colonisateur.

\section{Signification et portée sémantique des deux romans}

\section{Plan politique}

La quête du modernisme a amené l'étudiant vietnamien ayant séjourné plus de dix ans en France à introduire de nouvelles valeurs dans l'organisation socio-politique de son pays. Dans le roman Celui qui règnera, il fait remarquer:

Et pourtant, la pensée dévance l'homme. Déjà, et il y a de cela des siècles, Lao Tseu condamnait la propriété privée quand elle dépasse la norme des besoins: "Nul ne devant posséder et chacun pouvant utiliser, la propriété privée devient collective... Le communisme ou la propriété collective de la souche génératrice, ethnographique, de l'entité sociale que l'on nomme 
la commune, tous les membres de la souche étant égaux imprescriptiblement dans l'emploi et la jouissance du bien communal...."(78)

Comme on peut le constater, l'étudiant vietnamien rêve d'une société sans classes où les richesses nationales seront équitablement partagées. Son projet de société s'apparente au "communisme," doctrine politique qui prône une société égalitaire. Pourra-t-il balayer du revers de la main les coutumes millénaires et introduire de nouvelles valeurs susceptibles de donner plus de bonheur à son peuple? Les deux romans ne répondent pas totalement à cette interrogation. Ils laissent au lecteur un arrière goût d'inachevé. On ne sait pas, après lecture de Celui qui règnera et Frères de sang, qui du modernisme ou de la tradition sortira vainqueur de ce duel fracticide?

\section{Plan social}

La majorité des Vietnamiens ont compris le bien-fondé des réformes de l'étudiant. Cependant, certaines personnes qui jouissent des bienfaits de la tradition restent réfractaires au changement. Les deux romans ne proposent aucune issue pour concilier ces deux positions antagonistes. L'étudiant accusé d'avoir coupé la langue de Bay est conduit à la capitale pour purger sa peine et le peuple qui ne sait rien du "complot" pense que leur nouveau "roi," celui qui règnera, s'absente de façon momentanée. Déjà on se met à rêver. Un des villageois fait l'observation suivante dans le roman Celui qui règnera: "Notre village ayant abrité un roi, nous ne paierons plus d'impôts" (242). On peut d'ores et déjà affirmer que les idées de progrès, de modernisme de l'étudiant vietnamien qui connaissent déjà un réel engouement populaire vont survivre même si ce dernier diparaissait un jour. "Les hommes passent, les idées restent," telle peut être la leçon morale de cette oeuvre.

\section{Plan religieux}

Le héros-narrateur veut améliorer le quotidien de ses compatriotes mais il se garde de s'attaquer au fondement spirituel (le bouddhisme) de la société vietnamienne. Tous les changements sont possibles mais ceux qui touchent à la foi des individus sont plus difficiles à réaliser. D'ailleurs dans les romans Frères de sang et Celui qui règnera, il n'y a aucun passage qui indique que l'étudiant s'est converti à la religion chrétienne. Sa quête de modernité est plus sociale que spirituelle. Même s'il n'adhère pas à toutes les valeurs du bouddhisme, il sait que la religion est très 
importante pour l'équilibre psychologique de l'homme. C'est pourquoi il accepte l'idée de la construction d'une pagode, même s'il préfère dans son fond intérieur la construction d'une école.

\section{Conclusion}

Notre souci premier, tout au long de ce travail de recherche a été de montrer comment la littérature vietnamienne naviguait entre modernité et traditions et d'examiner également les rapports entre colonisateur et colonisé. Aussi, l'analyse du thème: "modernité et traditions" dans les romans Frères de sang et Celui qui règnera nous a permis de comprendre la complexité de ce sujet. En effet, le couple modernité et traditions, bien qu'étant antinomiques de nature sont obligées de vivre ensemble. De la même manière que les deux faces d'une même médaille. La modernité pourrait être perçue comme un bond que les hommes essaient de faire dans le futur et la tradition, une fidélité au passé.

- Le véritable problème posé dans les romans Frères de sang et Celui qui règnera se résume à la question de choix entre le passé et le futur. Tous les peuples développés ont été à un moment ou à un autre confrontés à cette problématique. L'Europe a connu son véritable essor économique au XVIII ème siècle (siècle des lumières) quand la science et la technologie ont occupé une place prépondérante dans la société. Le Japon, bien vrai que n'ayant pas renié ses traditions a envoyé au début du XIXème siècle les plus brillants de ses enfants à l'école Occidentale pour apprendre comme le dit Cheikh Hamidou Kane, dans l'Aventure ambiguë, "l'art de vaincre sans avoir raison." Aujourd' hui, le Vietnam, comme beaucoup de pays en voie de développement, se trouve en face de cette brûlante équation à savoir régler le "différend" entre modernité et traditions. Dans les romans Frères de sang et Celui qui règnera, l'écrivain ne propose aucune solution pour résoudre ce délicat problème. Il se contente de présenter le conflit traditions et modernisme. Et la fin du roman Celui qui règnera laisse un arrière gout d'inachevé au lecteur. La grande majorité des Vietnamiens adhèrent aux idées de modernisme mais l'initiateur de ce changement, l'étudiant Vietnamien, est emprisonné. En outre. le pouvoir de la tradition reste intact puisque rien n'indique dans les deux romans que les Vietnamiens ont décidé de tourner le dos à la piété filiale, a l'animisme, au confucianisme... Comme pour dire que le conflit traditions/ modernité n'est pas sur le point de prendre fin. Peut-être que les Vietnamiens devront suivre la voie japonaise à savoir trouver un "savant" dosage entre traditions et modernisme afin que les deux visions du monde 
puissent cohabiter sans tomber forcément dans des affrontements fratricides.

En réalité, la tradition et le modernisme sont complémentaires. La tradition peut être perçue comme l'ensemble des valeurs de civilisations d'un peuple. Elle englobe les mythes d'une société.

Le besoin de créer des mythes répond à un souci d'équilibre psychologique de l'homme: celui de prendre pour vrais nos fantasmes, nos craintes, nos aspirations, en un mot tous les sentiments enfouis dans notre inconscient qui nous aident à vivre et que nous n'arrivons pas toujours à exprimer de façon rationnelle. Partant de ce constat, on peut dire que la tradition participe à l'équilibre psychologique de l'homme tout comme le modernisme aussi. Nous ajouterons simplement que la diversité est facteur d'enrichissement et l'histoire de l'humanité prouve que les pays qui sont aujourd'hui les plus puissants du monde, à l'exemple des États-Unis sont ceux qui ont su s'ouvrir sur l'extérieur tout en gardant leur identité nationale.

Louisiana State University 


\section{Notes}

1. Albert Memmi, Le portrait du colonisé (Paris : Gallimard, 1985) 25.

2. Frantz Fanon, Les damnés de la terre (Paris : La Découverte, 1985) 27.

3. Memmi Albert, Portrait du colonisé (Paris: Gallimard, 1985) 26

4. Frantz Fanon, Les damnés de la terre, 36.

5. Jean-Paul Sartre dans la Préface de l'œuvre Portrait du colonisé d'Albert Memmi soutient que la colonisation a contribué à la déshumanisation du colonisé.

6. Albert Memmi, Portrait du colonisé, 10.

7. Cheikh Hamidou Kane: L'Aventure ambiguë (Paris : Julliard, 1961) 47.

8. Frantz Fanon dans son livre Les damnés de la terre dénonce l'iniquité du système colonial.

9. Linda Lê, écrivaine Vietnamienne, au cours d'un entretien accordé en avril 1999 au Magazine littéraire "Lire" prétend que l'écriture lui permet de s'exiler. (p.32)

10. Charles Baudelaire cité par Évelyne Cosset dans Les Fleurs du Mal (Paris: Nathan, 1996) 35.

11. Aimé Césaire, Cahier d'un retour au pays natal (Paris : Présence africaine, 1956) 9. 


\section{Works Cited}

Bokiba, André. Écriture et identité dans la littérature africaine. Paris: L'Harmattan, 1998.

Césaire, Aimé. Cahier d'un retour au pays natal. Paris: Présence africaine, 1956.

-. Discours sur le colonialisme. Paris: Présence africaine, 1962.

Chatenet, Jean. Petits Blancs, Vous serez tous mangés. Paris: Seuil, 1970. Cosset, Évelyne. Les Fleurs du Mal. Paris: Éditions Nathan, 1996.

Damas, Léon-Gontran. Pigments. Paris: Présence africaine, 1972.

Durand, Jean-François. Regards sur les littératures coloniales. Paris:

L'Harmattan, 1999.

Duras, Marguerite. L'Amant. Paris: Les Éditions de Minuit, 1984.

—. L'Amant de la Chine du Nord. Paris: Gallimard, 1991.

-. Un barrage contre le Pacifique. Paris: Gallimard, 1950.

Fanon, Frantz. Les damnés de la terre. Paris: La Découverte, 1985.

—. Peau noire, masques blancs. Paris: Seuil, 1996.

Kane, Cheikh Hamidou. L'Aventure ambiguë. Paris: Julliard, 1961.

Kristeva, Julia. Étrangers à nous-mêmes. Paris: Gallimard, 1991.

Memmi, Albert. Le Portrait du colonisé. Paris: Gallimard, 1985.

Pham, Van Ky. Celui qui règnera. Paris: Bernard Grasset, 1954.

—. Frères de sang. Paris: Seuil, 1947.

-. L'homme de nulle part: Légendes avec un hors-texte de Lê Phô. Paris: Éditions Frasquelles, 1946.

Ricoeur, Paul. Soi-même comme un autre. Paris: Seuil, 1996.

Said, Edward. Culture and imperialism. New York: Random House, 1993.

—. Orientalism. New York: Pantheon Books, 1978.

Suk, Jeanie. Postcolonial paradoxes in French Caribbean writing:

Césaire, Glissant, Condé. Oxford: Clarendon, 2001.

Winston, Jane Bradley. Postcolonial Duras: cultural memory in Postwar

France. New York: Palgrave, 2001.

Yeager, Jack Andrew. Vietnamese literature in French. New-Orleans:

Tulane University, 1999. 